\title{
El Pensamiento Sobre Asuntos Internacionales en el Caribe de Habla Inglesa El New World Group y su Herencia ${ }^{1}$
}

\author{
Eduardo Devés Valdés \\ Profesor e investigador del Instituto de Estudios Avanza- \\ dos de la Universidad de Santiago de Chile, Idea-Usach. \\ eduardo.deves@usach.cl<www.eduardodevesvaldes.cl>.
}

\section{Fernando Estenssoro Saavedra}

Profesor e investigador del Instituto De Estudios Avanzados de la Universidad de Santiago de Chile, Idea-Usach. fernando.estenssoro@usach.cl.

\section{Resumen}

Se trata de leer parte del pensamiento caribeño anglófono desde la pregunta por sus elaboraciones sobre asuntos internacionales y mundiales. El artículo se focaliza en la línea que une economía de plantación, dependentismo caribeño e integracionismo. Se divide en 3 partes: en la primera se presenta la noción "economía de plantación" en la segunda un conjunto de premisas para fundar un razonamiento sobre asuntos internacionales y mundiales, en la tercera las propuestas específicas: de integración y colaboración y sus variantes. La tesis que se argumenta es que las propuestas elaboran un marco teórico consistente con un criterio básico, la pequeña talla de los estados de la región. Por tanto, se excluye un conjunto de nociones clásicas de las relaciones internacionales, asumiendo otras razonables para las dimensiones y posibilidades de la región, donde la noción misma de "poder"

\footnotetext{
${ }^{1}$ Este artículo es parte del proyecto Fondecyt $\mathrm{N}^{\circ} 1110860$ : Hacia una cartografía del pensamiento internacionalista latinoamericano del siglo 20: obras, problemas, escuelas y categorías. Se agradece al personal de la biblioteca de la UWI, St. Agustin, Trinidad \& Tobago por la excelente atención dispensada. Todos los textos citados que en la bibliografía aparecen en inglés han sido traducidos por los autores.
} 
(y sus derivaciones) es casi irrelevante e incluso nociones como "margen de maniobra" y donde el quehacer de la diplomacia y la sociedad civil reemplaza completamente los agentes armados y la capacidad de manipular organismos internacionales.

Palabras-clave: Caribe. Integración caribeña. Economía de plantación. Pensamiento caribeño. New World Group.

\title{
INTERNATIONAL AFFAIRS THOUGHT IN THE ENGLISH-SPEAKING CARIBBEAN. THE NEW WORLD GROUP AND ITS LEGACY
}

\begin{abstract}
In this paper we try to understand the English-speaking thought of the Caribbean by asking ourselves the question about their elaborations on international and global affairs. The paper focuses on the links between the plantation economy, the Caribbean dependency and integrationism. The paper is divided into three sections: the concept of "plantation economy" is described in the first section; several premises, which are indispensable to support the reasoning on international and global matters, are presented in section 2; specific proposals regarding integration and collaboration and their combinations are made in the third section. The discussion of the thesis is that proposals provide for a consistent theoretical framework with one basic criterion, i.e. the small size of the states of the region. Some classic notions of international affairs are ruled out and are replaced by concepts that are far more consistent with the actual dimensions and possibilities of the region, in which the very notion of "power" (and its "derivatives") is virtually irrelevant. Or even notions such as "room for maneuver" where diplomacy and the civil society act as a substitute for armed agents and the ability to manipulate the international agencies.
\end{abstract}

Keywords: Caribbean. Caribbean integration. Plantation economy. Caribbean thought. New World Group.

\section{Sumário}

1 Introducción. 2 El tema de la economía de plantación. 3 Algunas premisas del pensamiento del New World Group y su entorno. 4 Propuestas. 5 Conclusiones. 6 Proyecciones. 7 Referencias. 


\section{INTRODUCCIÓN}

El sentido de este artículo, como parte de una investigación sobre pensamiento de América Latina Caribe acerca de asuntos internacionales y mundiales, consiste en relevar algunas ideas desarrolladas en el Caribe de habla inglesa, entre 1960 y 2010 , donde se articulan elementos como economía de plantación, dependencia, pequeña talla (small size), integración o colaboración y autonomía.

La hipótesis sobre la cual se sustenta este trabajo es que en el pensamiento del Caribe de habla inglesa se han desarrollado líneas de trabajo sobre asuntos internacionales y mundiales apuntando a formular criterios propios, en tensión con las corrientes principales de la disciplina, asumiendo como criterios fundamentales: la especificidad histórica y la pequeña dimensión de las economías y los estados, proponiendo como tarea fundamental formas de acción conjunta entre los estados y territorios, que ofrecerían mejores posibilidades.

En el ámbito de América Latina Caribe se ha ido haciendo cada vez más explícita la discusión en torno a la necesidad de formular propuestas acerca de asuntos internacionales y mundiales que sean coherentes con la situación de estos pueblos, teniendo en cuenta tanto condiciones específicas como sus especificidades culturales. La discusión acerca de elaboraciones teórico-conceptuales de la región y para la región ha ido asumiendo una importancia cada vez mayor en la medida que se ha consolidado una institucionalidad académica y diplomática. Argentina y Brasil, a este respecto, han sido los países más importantes (ver Devés, 2013, donde se entrega un estado de la cuestión), entre otros, pero también algunos de los países del Caribe de habla inglesa, y allí en primer lugar Jamaica y Trinidad \& Tobago cuentan con elaboraciones en este sentido. Las ideas sobre asuntos internacionales y mundiales son amplísimas e incluso si se considera sólo la producción en inglés es mucho mayor de 
lo que se mostrará aquí, donde no se trata de hacer un panorama, sino apenas focalizarse en el desarrollo de un tipo de pensamiento y para ello aludir a algunos antecedentes necesarios.

Sobre el pensamiento del Caribe de habla inglesa, expresado en las ciencias económico-sociales, se ha escrito bastante en tiempos recientes, especialmente acerca del New Worl Group (Millete, 2010; Girvan, 2006a; Benn, 2004; Devés, 2003, Conway; Timms, 2003: Blomstrom; Hettne, 1990; Sankatsing, 1989, entre otros) y sobre su pensamiento dependentista y en particular sobre la economía de plantación han escrito los mismos protagonistas (Girvan, 2010) y estudiosos de las ideas (Benn, 2004; Lewis, 1995; Sankatsing, 1989). Sin embargo, en tales trabajos no se ha puesto énfasis en la producción sobre asuntos internacionales y mundiales.

Ahora bien, estos desarrollos teóricos deben entenderse también como parte del cuestionamiento de lo que puede llamarse genéricamente el "euro-centrismo" o el "usa-centrismo" de los estudios internacionales. Por ejemplo, Fernando Galindo, ha planteado que "la disciplina de las Relaciones Internacionales tiene un marcado carácter occidental, europeo y norteamericano fundamentalmente" y como expresión de ello "tanto en el desarrollo de las agendas de investigación como en las categorías, los debates y las teorías predominantes, condiciones que evidentemente responden al 'nexo históricamente estrecho entre el poder (británico, europeo y estadounidense) y la producción de conocimientos” (2013, p. 87). Arlene Tickner y Ole Waever han destacado que, desde la descripción seminal realizada por Stanley Hoffmann en 1977 sobre las relaciones internacionales como una ciencia social estadounidense, ha sido común afirmar que las relaciones internacionales no son del todo "internacionales", sino que por el contrario, son bastante caracterizadas por la generalización de los modos de pensar anglo-americanos y sus respectivas fronteras conceptuales y espaciales (2013, p. 170). Ahora bien, las observaciones y críticas son en general, mucho mayores que las propuestas, escasas y 
formuladas rápidamente. Lo que los mismos autores definen al señalar que “ciertamente, algo bastante general acerca de este estado de la situación puede ser encontrado en la literatura existente en forma de crítica o de lamento" (2013, p. 168). B. Gruffydd Jones, por su parte, ha afirmado que "los puntos de referencia normales de la versión de la disciplina de las relaciones internacionales son casi completamente extraídos de la historia 'interna' europea. El canon disciplinario reconocido de las relaciones internacionales modernas consiste en el pensamiento europeo clásico" (2006, p. 2). En la misma línea, Melody Fonseca y Ari Jerrems plantean la necesidad de "un análisis de los orígenes eurocéntricos del pensamiento teórico de las relaciones internacionales, lo cual nos demuestra la necesidad de promover el enfoque decolonial dentro de las perspectivas críticas de la disciplina” (2012, p. 104).

Esta situación, denunciada por muchas personas que trabajan en los estudios internacionales en la actualidad, fue bastante clara para la intelectualidad del Caribe desde los 1960s. Dicho más ampliamente, para los estados del Caribe de habla inglesa, sería una suerte de esquizofrenia pensar sus relaciones internacionales o su inserción en el mundo con los criterios con que pretenden pensarse las grandes potencias, e incluso los grandes estados periféricos, o los países del Tercer Mundo con importantes recursos de materias primas (como es el caso de varios de los países de la América del Sur). Se asumió desde temprano que sus posibilidades estaban marcadas, en buena medida, por una cuestión de talla económica, geográfica y demográfica. De allí la necesidad de pensar de otro modo su quehacer y su inserción.

A continuación se desarrollan tres acápites: 1) el primero acerca del tema de la "economía de plantación" donde se presenta la definición de este género de economía-política, con las implicaciones que ello representa para los asuntos internacionales; 2) el segundo, sistematiza un conjunto de premisas que derivan del pensamiento del Caribe de habla inglesa, y 
que van a componer luego las propuestas sobre asuntos internacionales y mundiales; 3) en tercer lugar, se da cuenta de lo propuesto que, en síntesis, es un asunto de integración, aunque con variantes que derivan de matices y cambios en las formulaciones a lo largo del tiempo. Se termina con unas conclusiones y unas proyecciones a partir de este pensamiento que lo articulan al de otros lugares de América Latina Caribe.

Debe tenerse presente que se siguen muy de cerca las formulaciones de los propios autores, intentando armar sus planteamientos de manera sintética, apegándose a sus propias palabras.

\section{EL TEMA DE LA ECONOMÍA DE PLANTACIÓN}

La noción economía de plantación es una de las más originales y con mayores proyecciones del pensamiento del Caribe de habla inglesa. Esta noción, elaborada para describir y explicar el subdesarrollo de la región, fue entendida siempre como parte de un entramado teórico que concebía este género de economía y sociedad como parte de un sistema internacional, teniendo en cuenta factores naturales - dimensiones, ubicación geográfica, recursos - y humanos, tanto históricos como presentes.

La definición de una economía de plantación fue formulada por Lloyd Best y Karin Levitt en 1968: "Las frustraciones del programa de industrialización en el Caribe sólo pueden ser comprendidas a la luz del legado que los territorios han heredado de su común historia de plantación”. Incluso considerando ciertas diferencias, la "economía de plantación es un tipo específico de economía de hinterland: esta forma parte de la economía exterior de la metrópoli. Su papel consiste en producir materias primas para la venta en los mercados metropolitanos”, cosa que implica que "la fuente mayor de su capacidad empresarial, de sus finanzas 
e, incluso, el lugar de las decisiones clave son metropolitanas. El hinterland de plantación es esencialmente un área geográfica de producción pasivamente incorporada en la economía mundial” (2009b, p. 19).

Esta noción proviene de la necesidad de un modelo económico apropiado para el análisis del tipo de economía caribeña, especialmente dado el hecho que varios cientistas económico-sociales insatisfechos con los resultados de las políticas hasta allí implementadas, intentaban explicarse las razones de ello al señalar: "era nuestra profunda insatisfacción con las fracasos de las políticas de industrialización para reducir el sub-empleo, de postguerra. Una década de fuerte crecimiento económico en Jamaica en los 1960 no resultó en un crecimiento del empleo" (Best; Levitt, 2009a, p. 9). Por lo tanto, ello delataba que la estrategia se fundaba en "debilidades básicas del approach teórico de las políticas implementadas" y parecía que los diversos paradigmas en operación no eran adecuados para pensar-actuar en esa realidad, como bien planteaban estos autores:

$\mathrm{Ni}$ los modelos keynesianos de crecimiento ni tampoco el modelo ricardiano de los dos sectores, desarrollado por Arthur Lewis con la transferencia de trabajo desde el sector de subsistencia hacia el capitalista, fueron considerados apropiados para un análisis del tipo de economía prevaleciente en el Caribe. Las doctrinas prevalecientes estaban basadas en un modelo tomado de la experiencia histórica de los países industrializados.

Las economías del Caribe no son, ni han sido, economías 'duales'. Desde su origen, fueron creación de inversionistas aventureros de las metrópolis y el trabajo no-libre de los esclavos. Desde su creación, las economías del Caribe han sido operaciones ultramarinas, cuyo último control ha estado localizado en la metrópoli.

La tesis central de nuestro trabajo es que la economía caribeña ha tenido escaso cambio estructural, desde el establecimiento de la plantación esclavista hace más de 300 años (Best; Levitt, 2009a, p. 10-13). 
Cómo señaló Denis Benn, la crítica se focalizaba particularmente en la obra de Arthur Lewis, el más importante economista caribeño, que obtendría el Nobel de Economía en 1979, quien había proporcionado los elementos teóricos para el proyecto “industrialización por invitación”, que se estaba implementando durante los años 1960s. Tesis que habían sido reforzadas por el diagnóstico económico realizado por $\mathrm{W}$. Demas, durante los 1960s, quien "también proveyó justificación teórica para el approach de Lewis al desarrollo económico, argumentando que la pequeña talla de las economías del Caribe imponía límites a la capacidad de alcanzar crecimiento auto-sostenido”. Ello se debía, al igual que lo propuso la teoría del crecimiento económico de W. W. Rostow, popularizada como el "take off”, a que era necesaria "la generación de suficientes ahorros para financiar la inversión interna en la perspectiva de alcanzar la transformación estructural. La conclusión, entonces, fue que la dependencia de las inversiones de capital ultramarino eran virtualmente inevitables para tales economías" (Benn, 2004, p. 126).

Lewis había publicado en los 1950s, los trabajos Economic Development with Unlimites Supplies of Labour (1954) y la Theory of Economic Grows (1955). Estos fueron adoptados como la mejor propuesta para el desarrollo de la región. Lewis introdujo una forma modificada de las teorías neoclásicas, reinterpretando la teoría del desarrollo en el contexto de una ilimitada reserva de mano de obra. Su visión de las economías caribeñas como compuestas de un pequeño sector capitalista-moderno y un amplio sector tradicional de economía de subsistencia, demasiado improductivo para generar desarrollo, aunque capaz de sumistrar mano de obra barata para el sector industral que podría instalarse. Ahora bien, se suponía que usando la ventaja comparativa de mano de obra barata, las islas del Caribe podrían experimentar crecimiento económico y acumulación capitalista a través de la industrialización y que, en ese proceso, el sector agrario se modernizaría por las inversiones, la indutria toma- 
ría mano de obra del sector de subsistencia y se obtendrían suficientes recursos para importar los bienes industriales necesarios que no podrían producirse por el la pequeña talla de las economías.

Sin embargo, las propuesta de Lewis no había alcanzado los objetivos esperados, tal como los entendían los miembros del New World Group. Estos asumian que "la aplicación de las teorías de Lewis resultó en la continuación de la dependencia externa, el rápido crecimiento de la población urbana y desempleo, sin que el sector industrial cumpliera sus promesas, sumándose a ello la caída de la producción agrícola (ver Conway; Timms, 2002-2003, p. 97).

En vistas de una evaluación negativa que veía como fracasado el empeño de lograr el desarrollo, inspirándose en las teorías del crecimiento de Lewis (y W. W. Rostow), se trató de formular una propuesta de desarrollo autónomo a fin de obtener los resultados deseados. De esta forma, el nombre de Best quedó completamente asociado a la economía de plantación y a su crítica de Arthur Lewis. En este caso, no nos interesa presentar esta diferencia en relación a la caracterización de las economías del Caribe, si duales u originariamente capitalistas, sino que intentar la pregunta acerca de las implicaciones para una teorización de lo internacional. En este sentido, el New World Group, coincidiendo grosso modo con el planteamiento de la economía de plantación y la subsecuente crítica a la industrialización por invitación, planteó las cosas en términos de dependencia, antimperialismo y socialismo. Vale decir, mostraron una clara influencia de las ciencias económico-sociales generadas por aquella época en el Cono Sur de América Latina (ver Devés, 2003).

\section{ALGUNAS PREMISAS DEL PENSAMIENTO DEL NEW WORLD GROUP Y SU ENTORNO}

A continuación, se intentan sistematizar varias premisas que fueron cristalizando en el pensamiento del Caribe de habla inglesa y más específicamente al interior de sus ciencias económico-sociales. Estas aluden 
a ciertos principios "lógicamente" previos a la formulación de propuestas y que forman parte de los presupuestos, paradigmas dirían algunos, que se van asumiendo colectivamente, es decir, ciertos consensos, a partir de los cuales se elaboran propuestas de política exterior.

La formulación de estas premisas tiene por función exponer algunos criterios básicos que permiten entender el "contexto intelectual" en el cual se desarrollan los planteamientos del New World Group. Se elaboran brevemente tres de estas premisas: que es necesario un conocimiento correcto y de un quehacer intelectual pertinente, que existe una correlación entre factores epistémicos y factores psico-sociales, que la dependencia se encuentra asociada a la pequeña talla de las economías y a su balcanización. Estas permitirían sentar las bases de una teorización adecuada en cuestiones internacionales.

\section{Premisa 1: Que es necesario un conocimiento correcto y de un quehacer intelectual pertinente:}

El punto de partida que fundamentó esta preocupación fue el mal resultado de las políticas, que no alcanzaban los objetivos formulados. Ello no se atribuyó a la mala aplicación sino principalmente a un error en los conocimientos. Si no se entendía bien la realidad, malamente podía actuarse de manera eficiente. Para explicar por qué no se entendía cabalmente el funcionamiento de la realidad económico-social, G. Best y el New World Group señalaron el problema de la inadecuada formación, particularmente en economía, que se estaba impartiendo en la región. Si había una mala formulación se desprendían malas políticas. De allí el problema de la impotencia del Estado para gestionar la propia economía, cosa que supuestamente no entendían las escuelas: neoclásica, keynesiana y lewisiana. 
Diversos autores que se inspiran en las ideas del New World Group han agregado formulaciones a este afán de pertinencia: Denis Benn, Norman Girvan y Pedro Rivera. Pedro Rivera ha planteado que, en general, no se conocen, no se enseñan, los rudimentos de una economía caribeña, con sus diferencias y especificidades (ver Rivera, 2008). Denis M. Benn, ha destacado que el New World Group buscó un desarrollo intelectual independiente, "apropiado a la imaginación caribeña” (Benn, 2004, p. 123). Se trataba de entender las cosas en los propios términos, no adhiriendo acríticamente a discursos elaborados para otros pueblos y por otros pueblos. Como afirmó Girvan, la teoría de la economía de plantación destacaba la especificidad de la "experiencia caribeña," tratando de entenderla en sus propios términos, en oposición a los paradigmas provenientes de las metrópolis, es decir, "centrada en el Caribe más que eurocéntrica” (2009, p. XVII).

\section{Premisa 2: Que existe una correlación entre factores epistémicos y factores psico-sociales}

Este reclamo se halla en múltiples lugares del pensamiento del Caribe de habla inglesa y ha sido parcialmente sintetizado por E. K. Brathwaite al señalar que las deficiencias a nivel de la política exterior derivaría parcialmente de una "falta de confianza" que aparece basada psicológicamente en el factor histórico de la colonización y la orientación externa. El caribeño no reconoce su valor y la necesidad vital de obtener primacía internacional. Tales fuerzas "detrimentales" son generalmente "reforzadas por conflictos conceptuales, que redundan en falsa conciencia y episteme eurocéntrica (2006, p. 2). Entender la realidad y elaborar categorías propias fue una propuesta específica para el quehacer económico, aunque se proyectó primero hacia antecedentes históricos, entender la historia para entender la actualidad económica, y posteriormente hacia lo internacional, entender la economía afirmada en un conocimiento histórico para alcanzar mejor inserción internacional. Brathwaite afirmaba que, en consecuencia, 
numerosos teóricos descartan la ambivalencia del liberalismo y del realismo clásico porque "ambas teorías son incapaces de capturar las especificidades relevantes para los estado del Caribe y de los pequeños estados, en general”, teniendo en cuenta además que, el Caribe puede ser mejor entendido desde perspectivas que consideran las peculiaridades de las experiencias coloniales y postcoloniales derivadas de la específica relación del Caribe con el desarrollo histórico del orden global (2006, p. 6). El tema de la falta de autoconfianza se relaciona con la small size.

\section{Premisa 3 Que la dependencia se encuentra asociada a la pequeña talla de las economías y a su balcanización}

Sobre la small size han escrito numerosos autores, tratándose de un tema recurrente en diversos ámbitos del pensamiento: cuestiones económico-sociales, temas internacionales, ensayo sobre el carácter, entre otros. Girvan escribió "en el Caribe ha existido una conciencia aguda de las limitaciones impuestas por la pequeña talla” (1973, p. 8). William Demas (1987) ha sido uno de los que con mayor énfasis ha destacado y, quizás, el que ha otorgado más importancia a este factor, aludiendo a la talla de las economías como la variable principal de la dependencia, respondiendo a esta situación con la idea de regionalismo como el medio para ampliar el mercado doméstico y la base de recursos naturales, que ayudaría a superar las limitaciones de tamaño, y Clive Thomas, por su parte, ha asociado esto con los temas internacionales: "la talla impone una importante obstáculo al desarrollo regional, por lo que revertir la balcanización es esencial en la tarea por el desarrollo" (1988, p. 367).

\section{PROPUESTAS}

En la tarea de sistematizar el pensamiento sobre cuestiones internacionales del pensamiento del Caribe de habla inglesa y del New World Group se formulan aquellas propuestas que apuntan a formas acción con- 
junta de los estados del Caribe. Estas derivan de las nociones "economía de plantación”, "pequeña talla” y “dependencia”, teniendo en cuenta las premisas expuestas, la necesidad de un conocimiento correcto-pertinente, de la necesidad de elaborar categorías apropiadas para entender lo específico de estas realidades en el ámbito internacional y la dependencia, como condiciones que deben ser consideradas en la política internacional.

A continuación, se presentan varias propuestas que aparecen en el pensamiento del Caribe de habla inglesa y del New World Group, pretendiendo asumir sus condiciones con el fin de responder a los problemas o desafíos encontrados en el ámbito internacional. Todas estas pasan por formas de acción conjunta entre los estados de la región, sea económica y/política y/o cultural, bajo las formas de integración, cooperación o colaboración.

\section{Propuesta 1: Unión de los débiles para la defensa}

Una propuesta inicial, que luego ha tendido a desparecer, al menos en esta formulación del Girvan joven, conducía a pensar principalmente en términos de una suerte de unión de los países pobres para imponer condiciones a los poderosos. Esta propuesta fue claramente acentuada cuando se percibió el éxito en las acciones de la Organización de Países Exportadores de Petróleo. El poder de los productores de petróleo aparece como clave en la unión de países dependientes contra los ricos, es decir contra las compañías multinacionales. Al respecto, en 1975, Girvan señalaba: De repente, parece que las corporaciones multinacionales están a la defensiva. Los estados de la Organización de Países Exportadores de Petróleo han pateado las mesas de las gigantes compañías petroleras”. Y continuaba, "por primera vez, los países del tercer mundo están controlando activamente los términos del intercambio... Más aún, la acción de la Organización de Países Exportadores de Petróleo ha inspirado intentos similares de docenas de otros exportadores de materias primas” (1975, p. 26). D. 
Benn ha sostenido, comparando con la propuesta anterior de Lewis, la unión aduanera, que "el tipo de integración regional propuesto por Girvan y otros miembros del New World Group comprendía una genuina integración de las capacidades productivas regionales bajo el control de los gobiernos”, idea claramente diferente de la 'unión aduanera', como parte del programa de incentivos a la industrialización” (Benn, 2004, p. 149).

\section{Propuesta 2: Cooperación Gran Caribe}

Más tarde, y superando la noción de unión de los débiles para oponerse a los fuertes, Girvan ha destacado la distinción entre integración y cooperación. Según Girvan, una zona de cooperación puede ser definida "como un grupo de países que comparten un espacio común y un interés común derivado, que acuerdan cooperar (la Asociación de Estados del Caribe es un ejemplo de acuerdo de cooperación de este tipo)” (2006b, p. 20). Atribuye a esta cooperación un conjunto de tareas, tan prácticas como variadas, asociadas más a la pragmática cotidiana, y no solo con dimensión económica sino también ocupada de cuestiones como "desastres ambientales y naturales, medioambiente, salud y cultura, en las cuales la cooperación regional es relevante, especialmente en el caso de los pequeños países en desarrollo" (2006b, p. 45).

Sin abandonar las ideas sobre economía de plantación, sino que parcialmente reformulándolas y articulándolas a este proyecto, ${ }^{2}$ es decir, entendiendo la economía de plantación como fenómeno internacional, en 2008, Girvan planteó que "las economías que han funcionado bien en los años recientes son aquellas favorablemente dotadas para la expansión de

\footnotetext{
${ }^{2}$ Todas las economías caribeñas están en el mismo barco. "Por tanto, la teoría de la economía de plantación permanece tan relevante en la era de la globalización como lo fue en la etapa de la descolonización cuando surgió. Resulta evidente que la economía de plantación fue el producto de una forma anterior de la globalización; y la globalización actual es la etapa más reciente en la evolución de la economía metropolitana” (Girvan, 2008, p. XXI).
} 
nuevos productos de exportación, las que no lo están, permanecen empantanadas en la producción de los viejos productos". En este sentido, "romper o no el síndrome de la economía de plantación depende de cuánto un país es capaz de avanzar en el camino desde la incorporación pasiva a la activa en la economía internacional”. Esta ruptura consiste en abandonar un tipo de incorporación pasiva que "implica depender de la iniciativa y el espíritu de empresa metropolitanos y de una demanda externa exógenamente determinada” (2008, p. XXI). Para ejemplificar esta situación, Girvan señala:

Cuando los recursos se agotan o cambia la demanda externa, la economía retrocede. La incorporación activa involucra al empresariado local en la organización de la producción, en el acceso a la tecnología y el capital, y en la creación de mercados en el extranjero para la producción que no se puede vender internamente. Se forman vínculos inter-industriales y se invierten de manera local los excedentes. Así cuando los recursos naturales se agotan, o cambian la demanda externa, la economía puede asignar capital y fuerza de trabajo a nuevas actividades. Este es el desafío que enfrentan ahora las economías basadas en el turismo y la energía. Su florecimiento actual no puede ser eterno. Un reto similar enfrentan las economías estancadas (2008, p. XXI).

Sin embargo la tarea "estructural" si puede llamarse así, no desaparece completamente, pues postula que "la efectividad del regionalismo como estrategia para responder a la globalización, será una función del grado de su cooperación multi-funcional y de las relaciones extra regionales”. Es el caso de la “experiencia de la Asociación de Estados del Caribe que demuestra que el regionalismo puede ser una estrategia relevante en ausencia de la liberalización y la integración del mercado. En la esfera económica, el regionalismo puede complementar la integración bilateral y subregional” (2006b, p. 46).

\section{Propuesta 3: Producción-integración}

Otra de las propuestas de política internacional fue "producción-integración” o integración para mejorar la producción económica, idea que, según Richard Cato, “como un concepto estratégico, ha fascinado a 
los académicos y los agentes del desarrollo" (2005, p. 207). Esta noción ha sido elaborada por diversos autores en la trayectoria del pensamiento del pensamiento del Caribe de habla inglesa. Denis Benn y Kenneth Hall sostuvieron que "el concepto producción-integración representa un paradigma de desarrollo que ha sido objeto de una amplia teorización, tanto en el Caribe como en la comunidad internacional” (2006, p. XVI).

Cato (2005, p. 207) ha remontado los trabajos sobre el asunto a W. Demas, planteando que "de hecho en su estudio seminal de 1965 "Talla y desarrollo" se refería a producción integración como una tema relevante para el desarrollo económico de los países pequeños. Por su parte, Benn y Hall (2006, p. XVI) agregaron que esta extensa teorización puede remontarse hasta las tempranas formulaciones de A. Mc Intyre acerca de la 'combinación de recursos', que fue subsecuentemente construida por Brewster y Thomas en 1967, The dynamics of West Indian Economic Integration, en el cual señalaban que la integración de las West Indies no debería limitarse a las condiciones que gobiernan el intercambio de bienes, sino que debería incluir también la producción” (Cato, 2005, p. 207). Además, esta noción ha continuado vigente a través de las décadas porque más recientemente "un equipo dirigido por el profesor N. Girvan, preparó un extenso estudio acerca del tema en 1994, en el cual incluye consideraciones teóricas y prácticas para la promoción de la producción-integración” Cato (2005: 207). Más recientemente, Trevor Farrell ha publicado numerosos estudios acerca de la inversión transfronteriza en el Caribe, en los cuales el tema de producción-integración es muy relevante (Benn; Hall, 2006, p. XVI).

\section{Propuesta 4: Unión política para constituir un Estado-nación como Dinamarca o Suiza}

W. Demas propuso la creación de una unión política como respuesta a las necesidades y condiciones históricas. La condición histórico-cultural consistía en que el pueblo del Caribe de habla inglesa era una nación divi- 
dida en varias jurisdicciones y se trataba entonces de superar la small size, especialmente para llevar a cabo una mejor política exterior y alcanzar el buen gobierno. Para bregar en primer lugar con la talla económica necesitamos constituir un estado de un tamaño, razonable como Dinamarca o Suiza (1987, p. 83); a ello se sumaría "una política exterior y un designio común, y consecuentemente mayor fuerza y poder de negociación” y en tercer lugar y más importante "tal unión serviría para consolidar y dar completa forma institucional a nuestro sentido de identidad y fortalecer nuestra situación geopolítica”; cuarto, y siguiendo a Arthur Lewis, "la unión puede ayudar a asegurar un buen gobierno y la defensa de los derechos humanos" (Ibíd, p. 84). ${ }^{3}$

\section{Propuesta 5: La cuestión de una unidad cultural, el Mercado Común y la identidad}

Se ha dicho muchas veces que el tema de la acción conjunta de los estados del Caribe, no es únicamente económica, también comprende elementos políticos, sociales y culturales. En el pensamiento del Caribe de habla inglesa ha existido una línea de trabajo que ha puesto énfasis en estos asuntos. Por ejemplo, Alister Mc Intyre ha destacado la relación entre integración e identidad, lo que implica "volcarse desde el Mercado Común hacia las mayores dimensiones de la Comunidad”, porque es útil recordar que "la idea de integración ha sido puesta en relieve no solo por cuestiones relativas al desarrollo económico, sino también con los objetivos culturales y psicológicos de afirmar una identidad caribeña” (1987, p. 19). Afirma estas ideas ubicándolas en la trayectoria de los líderes de las independencias, destacando que "si se trata de identificar los elementos

\footnotetext{
${ }^{3}$ Las palabras de Lewis fueron: The fundamental reason for federating these islands is that it is the only way that good government can be assured to their peoples.
} 
comunes de los grandes líderes de los movimientos de independencia en la región, puede decirse que ellos más o menos compartieron a una imagen de la gente de las West Indies” (Ibíd.).

\section{Propuesta 6: La cuestión de una ideología para la integración}

Ian Boxill puso en relieve que si se quería desarrollar y sostener el sistema de integración regional era necesario guiarlo a partir de una "una ideología que promoviera el regionalismo" (1993, p. 29). Boxill pensaba que tal ideología debía ser desarrollada para construir un sistema económico y político que sostuviera el regionalismo y simultáneamente difundiera las ideas y valores de la integración”, de manera que el sistema “económico y político sería influenciado por esas actitudes y valores”. De este modo "la exitosa difusión de la ideología del regionalismo será reflejada en las relaciones entre los actores envueltos en el proceso de integración” (Ibíd.). Para Boxill, "una ideología del regionalismo debe reflejar una comunidad de percepción, explicación y acción en relación a la integración regional del pueblo. Especialmente "la necesidad de esa ideología se hace crítica cuando se reconoce que las evolución de la región hereda numerosos territorios con fuertes ligazones con países extra regionales" (Ibíd.).

\section{CONCLUSIONES}

1) Se ha mostrado una línea de reflexión que viene desde los 1960 s hasta la primera década del siglo 21 , donde se identifica un género de pensamiento internacional propio, elaborado sobre la base del modelo ampliado de economía de plantación y los conceptos que lo integran, particularmente "pequeña talla” y “dependencia”, y desde donde surgen propuestas de solución para obtener mayores niveles de autonomía, apelando, particularmente a nociones de políticas exteriores coordinadas, por parte de los estados caribeños. Por cierto, existen acentuacio- 
nes y hasta divergencias, aunque existe claridad y conciencia respecto de los elementos centrales. Se trata de un esquema obtenido a partir de trabajos de unas diez figuras que han incidido sobre estos temas, siendo Norman Girvan quien realiza mayor cantidad de aportes, a lo largo de las décadas y a lo ancho de los temas.

2) La idea clave en el pensamiento del New World Group acerca de temas internacionales apunta a fundar alguna forma de acción conjunta de los Estados, enfrentando el problema de la talla de las sociedades y sus economías y aprovechando cercanías y similitudes. En este sentido, el quehacer internacional no se conceptualiza como una manera de obtener poder, hegemonía o conseguir equilibrios estratégicos, sino por una parte, como defensa respecto de las compañías multinacionales y, por otra, como posibilidad de optimizar las formas de inserción en el espacio mundial, tanto como mejorar directamente la economía y/o los servicios y/o las condiciones de vida de las poblaciones. En este plano se produce una evolución entre los años 1960s-1970s y los 1990s-2000s, desde una colaboración para la defensa respecto de los poderosos hacia una colaboración para la inserción y el bienestar.

3) La noción Gran Caribe se construye como alternativa a la talla, en una reflexión acerca de la unión y sus variantes. Gran Caribe incorpora potencialmente a muchos estados, varios de los cuales no son insulares ni se conciben en primer lugar como caribeños, como es el caso de México, Colombia y Venezuela. La imagen “Gran Caribe” mejora el imaginario y permite presentarse mejor ante el mundo.

\section{PROYECCIONES}

1) La reivindicación de saberes alternativos o de la diferencia, se ha prestado para denunciar mucho y aportar poco o, si se prefiere: más para criticar lo hecho que para elaborar alternativas mejores. Esta orientación, 
sin embargo, no exime de asumir el problema y continuar avanzando en formulaciones sucesivas. Ahora bien, la capacidad de pensar desde el Sur o desde las periferias implica, entre otras cosas, insertarse en la trayectoria intelectual de esas mismas periferias. Es usual leer acerbas críticas al pensamiento del Norte hechas desde el mismo pensamiento del Norte, y que se mantienen al interior del supuesto círculo vicioso. Una de las maneras de romper con este es reconocer la trayectoria intelectual (relativamente) alternativa de las periferias y capitalizar allí (críticamente) lo posible para avanzar hacia nuevas formulaciones.

Una derivación perjudicial y obtusa de estas propuestas consistiría en justificar el desconocimiento de los estudios sobre el mundo o las relaciones internacionales que se realizan en el Norte. Es importante estudiar la teoría de las relaciones internacionales del Norte, aunque más para conocer cómo piensa el Norte que como teorías apropiadas para pensar el Sur.

2) Los aportes de las obras presentadas en este trabajo pretenden serlo, parcialmente por su originalidad, intentando pensar lo específico, o sea aquello que no ha sido pensado por otros o para otros, porque las condiciones han sido relativamente distintas. Ahora bien, ello no implica que lo que se ha presentado sea válido para todas las periferias, en todos los momentos y coyunturas. Se trata apenas de aportar otras miradas posibles y alimentar una reflexión, teniendo en cuenta que un gran número de los estados del mundo es de dimensiones e historias recientes similares a las del Caribe de habla inglesa y que, por tanto, este discurso puede ser más significativo que otros disponibles en los infinitos archivos del pensamiento.

\section{REFERENCIAS}

BENN, Denis M. The Caribbean An intellectual history 1774-2003. Kingston-Miami: Ian Randle Publishers, 2004. 
BENN, Denis; HALL, Kenneth. "Introduction”. In: CARICOM, Ian. Production integration. Kingston-Miami: Randle Publishers, 2006.

BEST, Lloyd; LEVITT, Karin. "A Historical and Institutional Approach to Caribbean Economic Development en Theory of Plantation Economy”, by Lloyd Best and Karin Levitt; University of the West Indies Press, 2009a.

BEST, Lloyd; LEVITT, Karin. "Outline of a general theory of Caribbean economy" en Theory of Plantation Economy, by Lloyd Best and Kari Levitt; University of the West Indies Press, 2009b.

BLOMSTOM, M.; HETTNE, B. La teoría del desarrollo económico en transición. México: F.C.E., 1990.

BOXILL, Ian. Ideology and Caribbean integration. Consortium Graduate School of Social Sciences, Mona Campus, Kingston: University of the West Indies, 1993.

BRATHWAITE, Edward Kamau. Contradictory Omens: Cultural Diversity and Integration in the Caribbean. Kingston, Jamaica: Savacou, 2006.

CATO, Richard. "Production integration: perspectives from the OECS". In: HALL, Kenneth; BENN, Denis. Caribbean Imperatives. Regional governance and integrated development. Kingston-Miami: Ian Randle Publishers, 2005.

CONWAY, Denis; BENJAMIN, Timms. "Where is the environment in Caribbean development theory and praxis?” In: Global Development Studies, vol. 3, n. 1-2, 2002-2003.

DEMAS, William. "Consolidating our independence: the major challenge for the West Indies”. In: MC INTYRE, Alister et al. Caribbean regionalism: challenges and options, Institut of International Relations, Trinidad and Tobago: UWI, 1987.

DEVÉS VALDÉS, Eduardo. “Cómo pensar los asuntos internacionales-mundiales a partir de la historia del pensamiento latinoamericano: Análisis de la teorización sobre relaciones internacionales”. In: Historia Unisinos, vol. 17, n. 1, 2013. Disponível em: <www.unisinos.br/revistas/index.php/historia/ article/download/.../1435 $\square$. 
DEVÉS VALDÉS, Eduardo El Pensamiento Latinoamericano en el Siglo XX. Entre la modernización y la identidad. Tomo II, Desde la Cepal al Neoliberalismo (1950-1990), Biblos - Dibam, Buenos Aires-Santiago, 2003. Disponível em: $<$ www.dibam.cl/publicaciones.asp?page $=2 \&$ catid $=77>$.

FONSECA, Melody; ARI, Jerrems. "Pensamiento decolonial: ¿una "nueva” apuesta en las relaciones internacionales?” In: Relaciones Internacionales, $\mathrm{n}$. 19, 2012.

GALINDO; Fernando. "Enfoques postcoloniales en Relaciones Internacionales: un breve recorrido por sus debates y sus desarrollos teóricos”, In: Relaciones Internacionales, Madrid: UAM, n. 22, feb./mayo 2013.

GIRVAN, Norman. "The development of dependency economics in the Caribbean and Latin America: Review and comparison”. In: Social and Economic Studies, Jamaica: UWI, v. 22, n. 1, march 1973.

. "Economic nationalists versus multinational corporations: revolutionary or evolutionary change?” In: AMIN, Samir; CARL, Winstrand. Multinational firm in Africa, Sweden Institute of African Studies, Upsala, 1975.

. "Caribbean Dependency Thought Revisited”. In: CJDS/RCED, vol. XXVII, n. 3, 2006a.

. Cooperation in the greater Caribbean: the role of the Association of Caribbean States, Kingston-Miami: Ian Randle Publishers, 2006b.

. La economía de plantación en la era de la globalización”. In: BEST, Lloyd; POLANYI-LEVITT, Kari. Teoría de la economía de plantación. Habana: Casa de las Américas, 2008.

. "Plantation economy in the age of globalization" in The theory of plantation economy, by LLOYD, Best; KARI, Levitt; Kingston: University of the West Indies Press, 2009.

. "New World and its critics" In: The thought of New World, Caribbean Reasonings, Editet by Brian Meecks and Norman Girvan , Ian Randle Publishers, UWI-Mona Jamaica, 2010. 
GRUFFYDD JONES, Branwen (Ed.). "From Eurocentrism to Epistemological Internationalism: power, knowledge and objectivity in International Relations". In: Decolonizing international relations, Londres y Boulder: Rowman \& Littlefield Publishers, 2006.

LEWIS, R. Walter Rodney's intellectual and political thought, Detroit: The Press of the West Indies-Jamaica y Wayne State University y Press, 1995.

McINTYRE, Alister. The Caribbean after Grenada four challenges facing the regional movements”. In: McINTYRE, Alister et al. Caribbean regionalism: challenges and options, Institut of International Relations, UWI, T\&T, 1987. MILLETE, James. "The New World Group: A historical perspective”. In: MEEKS, Brian; GIRVAN, Norman (Edit.). The thought of New World. The quest for decolonization. Kingston: Caribbean Reasonings, 2010.

RIVERA, Pedro. Teoría de la economía de la plantación. La Habana: Casa de las Américas, 2008.

SANKATSING, Glenn. Caribbean Social Science: An Assessment, Unesco, 1989. THOMAS, Clive Y. The poor and the powerless. Economic policy and change in the Caribbean, Londres: Latin American Bureau, 1988.

TICKNER, Arlene; OLE, Wæver. "Introducción: Epistemologías neo-culturales”. In: Review Relaciones Internacionales, n. 22, febrero/mayo 2013.

Recebido em: 17/12/2015

Aceito em: 17/12/2015 